\title{
Caracterização do tratamento medi- camentoso com estatinas em unidade básica de saúde
}

\section{Characterization of statin treatment in basic healthcare unit}

\author{
Mariana R. Bonfim , Acary S. B. Oliveira², Sandra L. Amaral ${ }^{3}$, Henrique L. Monteiro ${ }^{1,3}$
}

\begin{abstract}
RESUMO
Modelo do Estudo: Estudo retrospectivo. Objetivo do Estudo: Caracterizar as condutas frente às alterações lipídicas e efeitos colaterais durante o tratamento com estatinas em unidades básicas de saúde. Metodologia: Foram analisados os prontuários médicos de mulheres atendidas em uma unidade básica de saúde, dos quais foram obtidos: presença de doenças, medicamentos em uso, tipo e dose de estatina, resultados de exames bioquímicos, queixas osteomusculares, e cessação do uso da estatina, retroagindo as anotações até a consulta médica da prescrição inicial. Resultados: As estatinas prescritas foram sinvastatina e atorvastatina em doses baixas (10-20 mg). As modificações da dose $(48,4 \%)$ e/ou do tipo $(25,4 \%)$ ocorreram para adequação do perfil lipídico. Houve redução dos níveis lipídicos sem elevação dos valores de creatina quinase. A cessação de uso do medicamento (30,6\%) fol prioritariamente por conta própria (74\%), a qual apresentou forte associação com os relatos de desconfortos osteomusculares (Odds Ratio: 6,40[1,53-26,78]). Conclusão: A terapêutica com estatina foi eficaz para redução dos níveis séricos de lipídeos, e os autorrelatos de dor foram subestimados, caracterizando-se como o maior fator limitante da aderência ao tratamento.
\end{abstract}

Palavras-chave: Estatinas de HMG-CoA. Dislipidemias. Conduta do Tratamento Medicamentoso. Saúde Pública.

\section{Introdução}

As dislipidemias são caracterizadas por distúrbios no metabolismo de lipídeos, mais especificamente por alterações quantitativas de suas concentrações sanguíneas, como aumento nos triglicerídeos (TG), no colesterol total (CT), ácidos graxos livres (AGL), e Lipoproteína de Baixa Densidade (LDL-c), bem como redução na Lipoproteína de Alta Densidade (HDL-c). ${ }^{1}$
Reconhecida como um dos mais signficantes fatores de risco cardiovasculares, a dislipidemia isoladamente é responsável pelo desenvolvimento de $56 \%$ das doenças cardíacas e $18 \%$ dos casos de infarto, sendo ainda associada a um terço dos casos de mortalidade no mundo. ${ }^{2}$

Os inibidores da enzima 3-Hidroxi-3-Metilglutaril Coenzima A (HMG-CoA) redutase, também conhecidos como estatinas, são considerados os medicamen-
1. Programa de Pós Graduação em Ciências da Motricidade, Instituto de Biociências, Universidade Estadual Paulista (UNESP), Rio Claro, SP, Brasil.

2. Setor de Neuromuscular da Escola Paulista de Medicina, Universidade Federal de São Paulo (UNIFESP), São Paulo, SP, Brasil

3. Departamento de Educação Física da Faculdade de Ciências, Universidade Estadual Paulista (UNESP), Bauru, SP, Brasil
Correspondência: Mariana Rotta Bonfim Faculdade de Ciências, UNESP. Depto de Educação Física Av. Engenheiro Luiz Edmundo Carrijo Coube, 14-01 Bairro: Vargem Limpa. CEP: 17033-360 - Bauru/SP. Telefone: (14) 3103-6082 - Ramal: 7618. E-mail: mrb_unesp@yahoo.com.br

Artigo recebido em 11/05/2012 Aprovado para publicação em 06/11/2012 
tos de primeira escolha no tratamento dos distúrbios lipídicos, uma vez que seu uso reduz o LDL-c de $15 \%$ a $55 \%$, os TG de $7 \%$ a $28 \%$, e elevam o HDL-c de $2 \%$ a $10 \% .^{1,3}$ Tais modificações no perfil lipídico contribuem para a redução da mortalidade cardiovascular, da incidência de eventos isquêmicos coronários agudos e do acidente vascular cerebral, além de facilitar a revascularização do miocárdio. ${ }^{4}$

Apesar de bem tolerada pela maioria dos pacientes, a estatina está relacionada à ocorrência de efeitos tóxicos hepáticos e, principalmente, musculares. ${ }^{5}$ As lesões musculares decorrentes do uso das estatinas podem ser leves ou graves, variando desde a mialgia à rabdomiólise, e podem atingir cerca de $5 \mathrm{a}$ $10 \%$ dos pacientes. ${ }^{6}$ Os desconfortos musculares se manifestam como dores, câimbras e/ou rigidez muscular, além de redução de força muscular em idosos. ${ }^{5,6}$ O método preconizado para o diagnóstico e o acompanhamento da evolução destes desconfortos é a análise laboratorial dos níveis séricos da enzima creatina quinase (CK) juntamente com relatos clínicos, aspectos considerados pelo médico na conduta individual. ${ }^{3,7}$

De maneira geral, a eficiência e a relativa segurança demonstradas pelas estatinas tornaram esses medicamentos amplamente utilizados em todo o mun$\mathrm{do}^{1}$, sendo atualmente um dos mais comercializados. No ano de 2003, a atorvastatina alcançou o recorde de vendas de toda a história da indústria farmacêutica, sendo que o laboratório responsável pela sua comercialização obteve um lucro de 12,4 bilhões de dólares apenas em 2008. ${ }^{8}$ Apesar da ampla utilização da estatina, estudos apontam que a aderência dos pacientes ao tratamento é baixa, acarretando no não cumprimento das metas terapêuticas em muitos casos. ${ }^{9}$

Mauskop e Borden $(2011)^{10}$ destacam três diferentes razões que podem influenciar a aderência dos pacientes ao tratamento com estatinas: i) fatores dos pacientes, como condição socioeconômica, comorbidades e efeitos colaterais; ii) fatores médicos, como a aderência do profissional à aplicação das recomendações de diretrizes e interações com os pacientes; e, iii) do sistema de saúde, relacionados às questões de custo do medicamento e de acesso ao tratamento. Desta forma, considerando que as estatinas são amplamente utilizadas em nosso meio, faz-se relevante verificar como os fatores mencionados acima influenciam na prescrição, na aderência e na adoção de políticas públicas na atenção básica.

No Brasil, este fármaco é distribuído gratuitamente pelo sistema público de saúde e, por esta razão, pode-se supor que o fator relacionado ao sistema de saúde não seja representativo na aderência dos pacientes ao tratamento. Neste contexto, este trabalho teve por objetivo caracterizar as atitudes de mulheres usuárias de estatinas acompanhadas em uma unidade básica de saúde (UBS) e a conduta médica frente às alterações no perfil lipídico e aos efeitos colaterais decorrentes do uso deste fármaco.

\section{Material e Métodos}

O estudo retrospectivo foi conduzido a partir da análise dos prontuários de todas as mulheres usuárias de estatina, acompanhadas em uma unidade básica de saúde de uma cidade de médio porte do interior do Estado de São Paulo. O município em questão conta com dezessete UBS distribuídas na área urbana, tendo sido escolhida para este estudo a maior unidade da região central da cidade. Nesta unidade, a prescrição e o acompanhamento do tratamento dos pacientes acometidos pela dislipidemia são realizados de acordo com as diretrizes atuais, ${ }^{1}$ sendo o tratamento medicamentoso disponibilizado gratuitamente.

Inicialmente, foram identificados na unidade todos os usuários de estatinas. Numa etapa posterior, foram selecionadas apenas as mulheres, devido ao seu maior acometimento pelos desconfortos musculoesqueléticos decorrentes do uso do medicamento. ${ }^{6,7} \mathrm{~A}$ análise dos prontuários foi caracterizada pela busca de informações para responder a duas questões, a saber: i) situação atual de saúde da usuária; e ii) informações referentes ao histórico de uso da estatina.

Em relação à primeira questão, os dados foram obtidos a partir dos registros da última consulta médica, onde foram observados: a) os registros de outras doenças; b) as anotações de todos os medicamentos em uso, com atenção especial para aqueles com interação farmacológica potencial com as estatinas e aumentam o risco de efeitos colateriais, como fibratos, ciclosporina, antifúngicos, antibióticos do grupo macrolídeo, verapamil, inibidores da HIV protease, nefazodona e amiodarona; c) o tipo e a dose habitual de estatina; e d) relato de queixas osteomusculares.

Além disso, foram também obtidos dos prontuários os resultados do último exame bioquímico, sendo considerados apenas os realizados no máximo três meses antes da última consulta. Foram utilizados neste trabalho os parâmetros relacionados ao perfil lipídico (CT, LDL-c, HDL-c e TG), além dos biomarcadores de lesão muscular (Creatina Quinase [CK]) e hepáti- 
ca (Alanina Aminotransferase [ALT] e Aspartato Aminotransferase [AST]), sendo os resultados classificados em normais ou alterados ([CT $>200 \mathrm{mg}$ / $\mathrm{dL}$; [LDL-c $\geq 160 \mathrm{mg} / \mathrm{dL}$; [HDL-c $<50 \mathrm{mg} / \mathrm{dL}$; $[\mathrm{TG}>150 \mathrm{mg} / \mathrm{dL}] ;[\mathrm{CK}>170 \mathrm{U} / \mathrm{I}] ;[$ ALT $>31 \mathrm{U} /$ $\mathrm{mL}] ;$ [AST $>32 \mathrm{U} / \mathrm{mL}]){ }^{1}$

Com relação às condutas adotadas durante o tratamento com estatinas, a análise dos prontuários foi realizada retroagindo as anotações até a consulta médica de sua prescrição inicial; a partir deste momento, fez-se o registro das informações referentes ao perfil bioquímico, bem como do tipo e da dose de estatina primeiramente administrada, sendo ainda calculado o tempo total de uso. A partir desta consulta, realizou-se o levantamento das informações contidas nos prontuários visando identificar as condutas realizadas, sendo registrados: a) alterações no tipo ou na dose de estatina administrada e os motivos para a modificação (para redução mais acentuada nos parâmetros lipídicos; por ter atingido sua adequação; ou se para início de interações medicamentosas); b) episódios de cessação do uso, as causas motivadoras, e os relatos de reuso; e c) presença de desconfortos musculares, sendo identificados o tipo de queixa, os locais acometidos e a quantidade de consultas em que eles foram relatados.

Os dados obtidos foram categorizados considerando-se a presença/ausência de determinadas características, ou realizando grupamentos de acordo com a variável em questão. Para os dados categóricos, realizou-se a análise de frequência para identificar as características das usuárias das estatinas, o teste de qui-quadrado para verificar a existência de associações entre variáveis, e a regressão logística binária para indicar a magnitude das mesmas; os dados contínuos de perfil bioquímico foram apresentados como média e desvio padrão da média, e submetidos à análise não-paramétrica das diferenças entre duas medidas dependentes, utilizando o teste de Wilcoxon. Todas as análises foram realizadas por meio do software SPSS 17.0 for Windows, sendo adotado nível de significância de $\mathrm{p}<0,05$.

\section{Resultados}

\section{Situação atual de saúde das usuárias}

Foram identificadas na referida UBS 62 mulheres com dislipidemia que faziam uso regular de estatina (64,1 10,7 anos; peso: $69,5 \pm 14,3 \mathrm{~kg}$ ). Dentre as comorbidades apresentadas, a mais frequente foi hi- pertensão arterial sistêmica (83,9\%), seguida pelo diabetes mellitus (40,3\%), hipotireoidismo $(16,1 \%)$, artrite ou artrose $(4,8 \%)$, e fibromialgia $(1,6 \%)$.

$\mathrm{O}$ uso de outros fármacos além das estatinas foi documentado em $92 \%$ das mulheres, sendo que os anti-hipertensivos $(85,5 \%)$ e os hipoglicemiantes $(38,7 \%)$ foram os mais frequentes. Os medicamentos mais prescritos para o controle da pressão arterial eram da classe dos diuréticos $(45,2 \%)$, os inibidores da enzima conversora de angiotensina II $(38,7 \%)$ e os betabloqueadores $(33,9 \%)$; entre os hipoglicemiantes, a metformina foi o mais utilizado $(38,7 \%)$, seguido pelas sulfonilureias (6,5\%). Verificou-se ainda que $13 \%$ da casuística faziam uso de medicamentos que potencialmente interagem com as estatinas (fibratos [ $n=4]$, amiodarona $[n=2]$, antifúngicos $[n=1]$ e verapamil $[n=1])$.

A prescrição da sinvastatina foi feita em 78,7\% dos casos, enquanto que a atorvastatina em 21,3\% das mulheres. Em relação à dosagem diária, verificou-se que a dose mínima dos hipolipemiantes foi de $10 \mathrm{mg}$ (sinvastatina: $8,3 \%$; atorvastatina: $38,5 \%$; total: $14,8 \%$ ) e a máxima de $40 \mathrm{mg}$ (sinvastatina: $25 \%$; atorvastatina: $38,5 \%$; total: $27,9 \%$ ), sendo elevada a prescrição de doses de $20 \mathrm{mg}$ (sinvastatina: 64,6\%; atorvastatina: $23 \%$; total: $55,7 \%$ ). As queixas de desconfortos osteomusculares foram verificadas em nove $(14,5 \%)$ pacientes, sendo que as dores musculares ocorreram em seis e as articulares em três. No momento da última consulta, verificou-se número expressivo de mulheres com parâmetros alterados de CT $(41,7 \%)$, HDL-c $(71,2 \%)$ e TG $(44,3 \%)$, ao passo que os biomarcadores de lesão muscular e hepáticos aumentados apresentaram baixa ocorrência.

\section{Histórico de uso da estatina}

A partir da análise retroativa dos prontuários, foram identificadas 49 usuárias de estatinas que possuíam o registro da prescrição médica inicial do medicamento. Os dados indicaram que os medicamentos prescritos no início do tratamento foram a sinvastatina $(79,2 \%)$ e a atorvastatina $(20,8 \%)$; além disso, verificou-se que a prescrição das doses diárias eram de 10 mg (sinvastatina: $36,8 \%$; atorvastatina: $70 \%$; total: $43,8 \%$ ) e $20 \mathrm{mg}$ (sinvastatina: $63,2 \%$; atorvastatina: $30 \%$; total: $56,2 \%$ ).

Em relação ao tempo de uso do medicamento, verificou-se que seis pacientes foram indicadas a iniciar o tratamento na última consulta médica e, portanto, não possuíam histórico do uso. Em contrapartida, 
as demais mulheres $(n=43)$ apresentaram uma média de $22,9 \pm 18,9$ meses de uso de estatina, variando de três a 80 meses, aproximadamente. Considerando este intervalo, realizou-se a classificação das usuárias segundo tempo de uso: i) até 5,9 meses $(14,0 \%)$; ii) de seis a 17,9 meses (41,9\%); iii) de 18 a 29,9 meses (14,0\%); e iv) acima de 30 meses (30,2\%). Constatou-se que grande parte das usuárias de estatina encontrava-se em uso do medicamento entre seis a 18 meses.

O levantamento acerca das alterações no tipo do medicamento durante o tempo de uso indicou que uma parcela expressiva das mulheres $(25,6 \%)$ modificou o tipo de estatina administrada, sempre alternando entre sinvastatina e atorvastatina. Verificou-se, ainda, que para a maior parte das pacientes a dose primeiramente prescrita foi mantida $(51,2 \%)$, porém, para uma parcela expressiva delas houve aumento das doses $(41,9 \%)$. Nestes casos, a maior parte das pacientes recebeu a prescrição para adequação do perfil lipídico $(90,5 \%)$, sendo mínimo o número de reduções (7\%), as quais geralmente estavam associadas ao início do uso conjunto de fibrato (Figura 1A).

Ainda nessa perspectiva, as análises de quiquadrado indicaram presença de associação entre a modificação da dose do medicamento com a ocorrência de queixas osteomusculares $(\mathrm{p}=0,041)$, sendo que os resultados da análise estatística apresentados na Tabela 1 indicaram que as pacientes submetidas ao aumento nas doses de estatina tiveram quatro vezes mais chances de apresentarem queixas osteomusculares que aquelas que tiveram suas doses mantidas ou reduzidas durante o tratamento.

Com relação à cessação do tratamento, verificou-se uma frequência de aproximadamente $30 \%$, sendo a primeira ocorrência em média 5,3 $\pm 2,5$ meses após o início. Além disso, observou-se que a grande maioria das pacientes parou de tomar o medicamento por conta própria (74\%), sendo que os casos em que a cessação do uso foi parte da prescrição médica estavam associados ao início do uso de fibrato, à adequação do perfil bioquímico ou ao aumento de transaminases (Figura 1B). Neste último caso, a reintrodução da medicação não foi realizada, ao passo que em todos os outros ela foi novamente inserida, inclusive nos quadros de cessação por conta própria.

As queixas osteomusculares foram observadas em mais de $30 \%$ das pacientes, sendo as musculares mais frequentes que as articulares (Figura 1C). Nas alterações musculares, verificou-se que o local mais frequente da queixa eram os membros inferiores, ao passo que as articulares, em sua maioria, foram associadas aos dedos das mãos. Adicionalmente, a tabela 3 aponta que as pessoas que referiram queixas osteomusculares apresentaram 6,4 vezes mais chance de interromper o tratamento medicamentoso comparativamente aos não queixosos (Tabela 2).

\section{Tabela 1}

Distribuição da frequência de ocorrência de queixas osteomusculares de acordo com a modificação da dose do medicamento.

\begin{tabular}{lccc}
\hline & \multicolumn{2}{c}{ Queixas Osteomusculares } & \\
\cline { 2 - 3 } Dose de Estatina & Presente (\%) & Ausente (\%) & Taxa de Odds (IC95\%) \\
\hline Aumento & $9(50,0)$ & $9(50,0)$ & \multirow{4}{*}{, $\mathbf{0}(\mathbf{1 , 0 4 - 1 5 , 3 8 )}$} \\
\cline { 2 - 3 } Redução/Manutenção & $5(20,0)$ & $20(80,0)$ & \\
\hline
\end{tabular}

\section{Tabela 2}

Distribuição da freqüência de interrupção do tratamento de acordo com a ocorrência de queixas osteomusculares.

\begin{tabular}{|c|c|c|c|}
\hline \multirow[b]{2}{*}{ Queixas Osteomusculares } & \multicolumn{2}{|c|}{ INTERRUPÇÃO do TrataMENTO } & \multirow[b]{2}{*}{ Taxa de Odds (IC95\%) } \\
\hline & $\operatorname{Sim}(\%)$ & Não (\%) & \\
\hline Presente (\%) & $8(57,1)$ & $6(42,9)$ & \multirow{2}{*}{$6,40(1,53-26,78)$} \\
\hline Ausente (\%) & $5(17,2)$ & $24(82,8)$ & \\
\hline
\end{tabular}



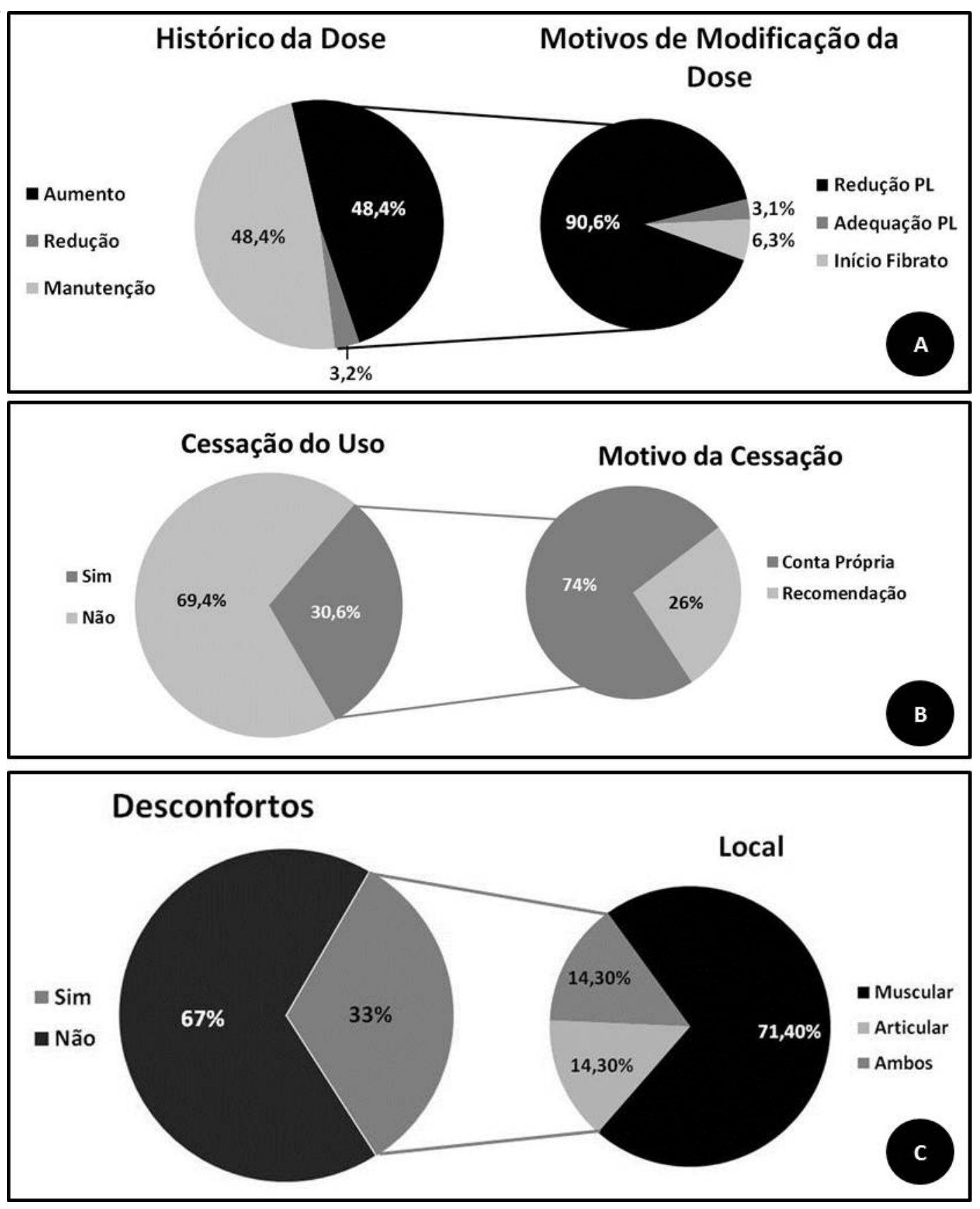

Figura 1. Características do histórico do uso de estatina. Legenda: PL = Perfil Lipídico.

Quanto aos valores dos exames bioquímicos relacionados à primeira prescrição de estatina, verificou-se que o parâmetro do perfil lipídico mais frequentemente alterado era o CT, seguido pelo HDL-c, TG e LDL-c. Com relação aos marcadores de lesão muscular, verificou-se que 10 mulheres apresentaram valores de CK alterados $(17,1 \%)$ e, destas, 7 (12\%) também apresentaram alteração dos marcadores de le- são hepática (AST e ALT). Os resultados da análise de qui-quadrado apontaram que a escolha pelo tipo de estatina, bem como pelas doses prescritas não apresentou associação com os parâmetros lipídicos que se encontravam alterados no início do tratamento $(\mathrm{p}>0,05)$.

A análise da evolução dos parâmetros bioquímicos, para o qual se considerou os valores obtidos na 
data de início do uso do medicamento e aqueles da última consulta médica, indicou que apenas o colesterol total apresentou alteração significante, tendo seus níveis reduzidos (Tabela 3). Em contrapartida, verificou-se que o tratamento administrado pelas mulheres do estudo não elevou os níveis dos biomarcadores de lesão muscular e hepática, sendo que apenas uma pequena parcela apresentou valores alterados no último exame.

\section{Discussão}

Expressiva parcela das pacientes dislipidêmicas era acometida por outras comorbidades, sendo a hipertensão arterial e a diabetes mellitus as mais frequentes. De maneira semelhante, Turi et al (2011) ${ }^{11}$ verificaram, em amostra composta por 222 usuários de duas UBS de Bauru, que 59,9\% possuia hipertensão e diabetes, dos quais, 31,5\% eram também acometidos por hipercolesterolemia. Além disso, Menezes et al (2011) $)^{12}$ observaram frequências elevadas de hipertensão arterial e dislipidemia entre pacientes diabéticos usuários de "Academias da Cidade" de Belo Horizonte; estas investigações demonstram ser forte associação entre essas doenças.
A terapêutica recomendada para o tratamento destas desordens envolve estratégias de tratamento medicamentoso e não medicamentoso, dependendo de cada caso e dos fatores de risco envolvidos na gênese das doenças. ${ }^{13}$ Quanto ao tratamento medicamentoso, os dados da presente pesquisa corroboram com as recomendações acima, uma vez que demonstram que mais de $90 \%$ faziam uso de estatinas associadas a outro medicamento. Apesar da necessidade do tratamento das comorbidades, atenção especial deve ser atribuída às interações medicamentosas, uma vez que alguns fármacos podem afetar o metabolismo das estatinas resultando no aumento de sua concentração e, portanto, acarretando em risco aumentado da manifestação de efeitos colaterais. ${ }^{14}$

Nesta investigação, verificou-se que apesar do grande número de medicamentos administrados aos pacientes, a frequência de uso dos fármacos que interagem com as estatinas foi baixa, sendo mais frequente o uso conjunto com fibratos. A esse respeito, as diretrizes nacionais apontam que casos de rabdomiólise podem ocorrer devido à associação de estatinas e fibratos, recomendando evitá-la ou prescrevê-la com cautela. ${ }^{1} \mathrm{Na}$ presente pesquisa, verificou-se que nos casos onde esta associação se fez necessária, a con-

\section{Tabela 3}

Evolução dos parâmetros bioquímicos (mg/dL) das usuárias de estatinas.

\begin{tabular}{|c|c|c|c|c|c|}
\hline \multirow[b]{2}{*}{$\begin{array}{l}\text { Parâmetros } \\
\text { Bioqímicos }\end{array}$} & \multicolumn{2}{|c|}{ INICIAL } & \multicolumn{2}{|c|}{ Atual } & \multirow[b]{2}{*}{$\mathbf{p}$} \\
\hline & Média $\pm \mathrm{DP}$ & $\begin{array}{c}\text { Parâmetros } \\
\text { Alterados }(\%)\end{array}$ & Média $\pm D P$ & $\begin{array}{c}\text { Parâmetros } \\
\text { Alterados (\%) }\end{array}$ & \\
\hline CT $(n=45)$ & $234,0 \pm 41,1$ & 78,3 & $197,8 \pm 48,1$ & 41,7 & 0,002 \\
\hline HDL-c $(n=42)$ & $47,7 \pm 12,7$ & 59,1 & $44,74 \pm 10,0$ & 74,5 & 0,079 \\
\hline LDL-c (n=37) & $146,7 \pm 32,7$ & 29,3 & $123,2 \pm 37,5$ & 18,2 & 0,055 \\
\hline TG $(n=45)$ & $180,8 \pm 86,1$ & 56,5 & $156,5 \pm 89,2$ & 43,8 & 0,157 \\
\hline CK $(n=28)$ & $104,3 \pm 57,1$ & 17,1 & $111,2 \pm 61,8$ & 23,1 & 0,848 \\
\hline $\operatorname{AST}(\mathbf{n}=27)$ & $20,7 \pm 5,9$ & 9,1 & $20,7 \pm 9,9$ & 7,5 & 0,675 \\
\hline $\operatorname{ALT}(\mathbf{n}=27)$ & $22,7 \pm 11,9$ & 11,8 & $19,8 \pm 10,7$ & 5,0 & 0,709 \\
\hline
\end{tabular}

Legenda: $\mathrm{CT}$ = Colesterol Total; HDL-c = Lipoproteína de Alta Densidade; LDL-c = Lipoproteína de Baixa Densidade; TG = Triglicérides; CK = Creatina Quinase; AST = Aspartato Aminotransferase; ALT = Alanina Aminotransferase.

Nota: Para fins de análise estatística, foram considerados apenas os pacientes que apresentavam no prontuário clínico os resultados iniciais e finais dos parâmetros. 
duta adotada foi de redução da dose de estatina para dar início à terapêutica conjunta com fibratos, demonstrando cautela na prescrição.

Em contrapartida, para uma parcela expressiva das mulheres, verificou-se a necessidade de aumento das doses do medicamento durante o tratamento, bem como da modificação do tipo de estatina, visando maior adequação dos níveis lipídicos. Estas ocorrências podem estar relacionadas às baixas doses prescritas no início do tratamento, bem como à limitação dos tipos de estatina prescritas. No município em questão, a disponibilidade gratuita de estatina pelo sistema de saúde se restringe à sinvastatina e atorvastatina, classes de alto e intermediário risco para ocorrência de alterações musculares. ${ }^{15,16}$ Assim, considerando que a ocorrência de miopatia por estatinas é dosedependente, ${ }^{1,7}$ a prescrição inicial e a manutenção de baixas dosagens durante o tratamento podem ser consideradas condutas preventivas ao desenvolvimento destes efeitos colaterais.

A esse propósito, apesar da ausência de associação entre o tipo de estatina prescrita no início do tratamento e o parâmetro lipídico alterado, observouse redução significante nos valores de colesterol total. Além disso, apesar da redução dos valores de LDLcolesterol ter apresentado significância marginal, o que pode estar relacionado ao número reduzido da amostra, os resultados apresentados indicam que as medidas terapêuticas adotadas foram eficazes no controle dos quadros de dislipidemia. Esta assertiva é ainda reforçada pelas baixas frequências de ocorrência de alterações dos parâmetros hepáticos e musculares, o que se faz extremamente relevante, visto que os raros, porém possíveis efeitos colaterais das estatinas acometem predominantemente estes tecidos. . $^{13,5,17-20}$

Como alternativa para reduzir o risco de intolerância à estatina, recomenda-se o monitoramento da terapêutica pela obtenção inicial dos parâmetros lipídicos, bem como das transaminases e creatina quinase, os quais devem ser reavaliados semestralmente. ${ }^{1,3,17,18}$ Os dados obtidos a partir dos prontuários clínicos dos pacientes demonstrou que este procedimento vem sendo realizado dentro da UBS avaliada, uma vez que os exames solicitados para avaliar os parâmetros lipídicos eram sempre acompanhados pela avaliação de transaminases e CK. A partir destes parâmetros, as condutas a serem tomadas no tratamento podem ser ponderadas - assim, recomenda-se o monitoramento dos usuários que apresentem elevações de 1 a 3 vezes o limite superior da normalidade nas tran- saminases, ${ }^{1,17-20}$ ou de 3 a 7 vezes na $C K,{ }^{1,7,17-20}$ ao passo que valores acima de 3 e 10 vezes esse limite, respectivamente, requerem a suspensão do medicamento. ${ }^{1,7,17-20}$

Apesar dessas recomendações, ainda há muita controvérsia no meio clínico e científico quanto ao uso da CK na identificação das lesões musculares por estatinas. Isto porque, em muitos casos, o paciente pode apresentar CK aumentada com ausência das manifestações clínicas, ${ }^{21}$ como também apresentar as manifestações clínicas e possuir valores normais de CK. ${ }^{7}$ De fato, verificou-se neste estudo que grande parte das mulheres apresentou queixas osteomusculares durante o período de tratamento com estatinas; e ainda, que a presença destas queixas se associou de forma significativa ao aumento da dose do medicamento, podendo ser considerada fator de risco para interrupção do uso do medicamento por conta própria.

Estes dados demonstram que, apesar da ausência de valores de CK acima de 10 vezes os valores normais, sendo desnecessária a interrupção do uso do medicamento, as manifestações clínicas foram decisivas para a aderência do paciente ao tratamento. Tem-se observado que os desconfortos mais comuns relacionados ao uso de estatinas se apresentam como dores, câimbras e/ou rigidez muscular, ${ }^{16}$ além da redução da força, ${ }^{21}$ problemas estes que interferem negativamente na realização das atividades diárias. ${ }^{6,7,16}$ Sobre estes dados, Thompson et al. ${ }^{7}$ ressaltam que o auto-relato de dor pelo paciente deve ser levado em consideração tanto quanto os valores de CK, sendo que ambas as situações devem ser consideradas indicativos de lesão muscular por estatinas.

Os relatos de desconfortos musculares utilizados neste estudo foram obtidos diretamente das anotações contidas no prontuário clínico do paciente e, em alguns casos, dado o caráter multifatorial destas queixas, podem não apresentar relação causal com o uso da estatina, o que pode ser entendido como uma limitação do estudo. Entretanto, se considerada a forte associação entre estes relatos habituais na atenção básica e a cessação do uso do medicamento por conta própria observada na presente pesquisa, pode-se inferir que as ocorrências de efeitos colaterais musculares pelas estatinas na prática clínica estão subestimadas, apontando para a necessária atenção aos relatos individuais de dor.

Tomados em conjunto, os resultados deste estudo sugerem que o tratamento com estatina no sistema de saúde pública de Bauru/SP é realizado predo- 
minantemente com sinvastatina e atorvastatina em doses baixas, sendo as modificações de dose e/ou do tipo de medicamento definidas principalmente pela adequação do perfil lipídico. Apontam, ainda, que esta medida terapêutica reduz os níveis lipídicos sem elevar substancialmente os valores de creatina quinase, sendo desnecessária a recomendação da suspensão do medicamento, caracterizando-se como eficaz. Em contrapartida, observou-se elevada ocorrência de cessação de uso do medicamento por conta própria, a qual apresentou forte associação com os relatos de desconfortos osteomusculares, demonstrando que esta relação é ainda subestimada. Estes dados também indicam que possivelmente o maior fator limitante da aderência ao tratamento com estatinas na saúde pública seja os efeitos colaterias vivenciados pelos pacientes, demonstrando assim a necessidade de dispensar maior atenção aos seus auto relatos cotidianos de dor.

\section{Agradecimentos}

Agradecemos a Secretaria de Saúde e a equipe do Núcleo de Saúde "Vila Cardia" do município de Bauru/SP pela disponibilização do material utilizado nesse trabalho e pela atenção dispensada.

\section{Financiamento}

Apoio financeiro: CAPES

\begin{abstract}
Study Model: Retrospective study. Study Objective: To characterize statin treatment management due to lipid alterations and side effects throughout statin treatment in basic healthcare unit. Methods: Medical reports of women from a basic healthcare unit were analyzed, obtaining: disease presence, regular medication prescription, statin type and dosage, biochemical exams results, musculoskeletal complaints, and statin use cessation, going back the information until the medical consultation of first prescription. Results: Prescribed statins were Simvastatin and Atorvastatin at low doses (10-20 mg). Dose $(48,4 \%)$ and/or type $(25,4 \%)$ alterations occurred for lipid profile adequacy. Lipid levels were reduced without creatine kinase elevation. Treatment withdrawn $(30,6 \%)$ was mainly due to their own decision (74\%), which was strongly associated with records of musculoskeletal complaints (Odds Ratio: 6,40[1,5326,78]). Conclusion: Statin treatment was effective in reducing serum lipid levels and self-reported pain was underestimated, characterizing the major limiting factor for treatment adherence.
\end{abstract}

Key-words: Statins, HMG-CoA. Dyslipidemias. Medication Therapy Management. Public Health.

\section{Referências}

1. Sociedade Brasileira de Cardiologia. IV Diretrizes brasileira sobre dislipidemias e prevenção da aterosclerose. Arq Bras Cardiol. . 2007;88:2-19.

2. World Health Organization. The world health report 2002: reducing risks, promoting healthy life. Geneva: World Health Organization, 2002. [Acessado em 20 de Março de 2012]. Disponível em http://www.who.int/whr/2002/en/.

3. Pasternak RC, Smith SC Jr, Bairey-Merz CN, Grundy SM, Cleeman JI, Lefant C. American College of Cardiology. ACC/ AHA/NHLBI clinical advisory on the use and safety of statins. J Am Coll Cardiol. 2002;40:567-72.

4. Goldenberg N, Glueck C. Efficacy, effectiveness and real life goal attainment of statins in managing cardiovascular risk. Vasc Health Risk Manag. 2009;5:369-376.

5. Brown WV. Safety of statins. Curr Opin Lipidol. 2008; 19:55862.

6. Joy TR, Hegele RA. Narrative review: statin-related myopathy. Ann Intern Med. 2009;150:858-68.
7. Thompson PD, Clarkson PM, Rosenson RS. An assessment of statin safety by muscle experts. J Am Coll Cardiol. 2006;97(8A).

8. Pfizer. Doing this differently. 2008. [Acessado em 15 de Março de 2012]. Diponível em http://www.pfizer.com/files/ annualreport/2008/annual/review2008.pdf.

9. Wong MCS, Jiang JY, Griffiths SM. Adherence to lipid-lowering agents among 11,042 patients in clinical practice. Int $\mathrm{J}$ Clin Pract. 2011;65:741-8.

10. Mauskop A, Borden WB. Predictors of statin adherence. Curr Cardiol Rep. 2011;13:553-8.

11. Turi BC, Codogno JS, Fernandes RA, Monteiro HL. Associação entre doenças crônicas em adultos e redução dos níveis de atividade física. Medicina (Ribeirão Preto). 2011;44:389-95.

12. Menezes MC, Pimenta AM, Santos LC, Lopes ACS. Fatores associados ao diabete mellitus em participantes do programa 'Academia da Cidade' na região leste do município de Belo Horizonte, Minas Gerais, Brasil, 2007 e 2008. Epidemiol Serv Saúde. 2011;20:439-48. 
13. Sociedade Brasileira de Cardiologia. I Diretiz brasileira de diagnóstico e tratamento da síndrome metabólica. Arq Bras Cardiol. . 2005;84(Supl 1).

14. Venero CV, Thompson PD. Managing statin myopathy Endocrinol Metab Clin North Am. 2009;38:121-36.

15. Rallidis LS, Fountoulaki K, Anastasiou-Nana M. Managing the underestimated risk of statin-associated myopathy. Int $\mathrm{J}$ Cardiol. 2011. In Press.

16. Bruckert E, Hayem G, Dejager S, Yau C, Bégaud B. Mild to moderate muscular symptoms with high dosage statin therapy in hyperlipidemic patients - the PRIMO study. Cardiovasc Drugs Ther. 2005;19:403-14.

17. Third report of the National Cholesterol Education Program (NCEP) Expert Panel on detection, evaluation, and treatment of high blood cholesterol in adults (Adult Treatment Panel III): final report. Circulation. 2002;106:3143-421.

18. Reiner Z, Catapano A, Backer G, Graham I, Taskinen MR, Wiklund O, et al. ESC/EAS guidelines for the management of duslipidaemias: the task force for the management of dyslipidaemias of the European Society of Cardiology (ESC) and the European Atherosclerosis Society (EAS). Eur Heart J. 2011;32:1769-818.

19. Genest J, McPherson R, Frohlich J, Anderson T, Campbell N, Carpentier A, et al. 2009 Canadian Cardiovascular Society/ Canadian guidelines for the diagnosis and treatment os dyslipidemia and prevention of cardiovascular disease in the adult - 2009 recommendations. Can J Cardiol. 2009;25:56779.

20. Mancini GBJ, Baker S, Bergeron J, Fitchett D, Frohlich J Genest J, et al. Diagnosis, prevention and management of statin adverse effects and intolerance: proceedings of a Canadian working group consensus conference. Can J Cardiol. 2011;27:635-62.

21. Glueck CJ, Rawal B, Khan NA, Yeramaneni S, Goldenberg N, Wang $P$. Should high creatine kinase discourage the initiation or continuance of statins for the treatment of hypercholesterolemia? Metab. 2009; 58:233-8.

22. Krishnan GM; Thompson PD. The effects of statins on skeletal muscle strength and exercise performance. Curr Opin Lipidol. 2010;21:324-328. 